\title{
EnkephaloVision: Anatomical Functionality Indicated by Ultrashort Transient Regional EEG Spectral Power Changes during Cognitive and Emotional Challenges
}

\author{
Wilfried Dimpfel ${ }^{1}$, Gwladys Nina Chiegoua Dipah ${ }^{2}$, Nigel Gericke ${ }^{3}$ \\ ${ }^{1}$ Justus-Liebig-University, Giessen, Germany \\ ${ }^{2}$ NeuroCode AG, Wetzlar, Germany \\ ${ }^{3}$ HG \& H Pharmaceuticals (PTY) Ltd., Bryanston, South Africa \\ Email: dimpfel1945@web.de
}

Received 15 March 2016; accepted 1 May 2016; published 4 May 2016

Copyright (C) 2016 by authors and Scientific Research Publishing Inc.

This work is licensed under the Creative Commons Attribution International License (CC BY).

http://creativecommons.org/licenses/by/4.0/

(c) (i) Open Access

\begin{abstract}
Anatomical functionality is a major topic in brain research. Numerous investigations have shown task dependent activation of focal brain areas, with most information based on time-averaged data due to methodological limitations. Ultra-fast quantitative EEG, especially in the newly developed combination with eye tracking (EnkephaloVision), is very suitable to follow activities of local electric circuits. This investigation in 57 subjects revealed transient focal frequency changes reaching up to more than $6000 \%$ of global median spectra power during cognitive and emotional challenges at frontal electrode positions. Recording epochs of $364 \mathrm{~ms}$ uncovered coherences with respect to focal brain areas and single frequencies, which are typically lost during averaged calculations. When averaging data over a whole scene, a least demanding challenge like viewing a boring animal video only activated the lateral frontal lobe, whereas solving brain-teasers and performance of mathematical calculations led to delta (modulated by acetylcholine) and theta (modulated by norepinephrine) increases in all brain regions in a statistically significant manner. In addition to delta and theta increases, performance of the Stroop test led to beta2 (related to GABAergic transmission) increases in the temporal lobe. The higher the mental demand the more brain regions were involved during 10 different challenges. There was no challenge which did not activate the lateral frontal brain in terms of increases of delta and theta spectral power. The results are in line with the view that the lateral frontal lobe is involved primarily during cognitive and emotional behavior related to activity changes of acetylcholine and norepinephrine.
\end{abstract}

\section{Keywords}

EnkephaloVision, Quantitative EEG, Spectral Power, Eye-Tracking, Cognition, Emotion, CATEEM 


\section{Introduction}

There is a longstanding debate on anatomical functionality. Experimental studies based on use of Nuclear Magnetic Resonance (NMR) and Positrons Emission Tomography (PET) suggest that particular anatomical structures are more involved during cognitive (for example frontal lobe) and emotional behavior (for example amygdala) than others. For example, the Anterior Temporal Lobe (ATL) has been implicated in numerous functional domains, including language, semantic memory, social cognition, and facial identification and it was found that the left lateral ATL is interconnected with hubs of the temporo-sylvian language network [1]. On the other hand, there is ample evidence that cognitive and emotional processes relate to electric network activity involving several anatomic regions of the brain. The communication structure between these regions is based on electrochemical processes. Neurotransmitter activity activates ionic channels leading to changes in electric behavior of neurons. Indirect measurements of neuronal activity as achieved by NMR techniques only provide evidence of more or less activation of particular Regions of Interest (ROI). Direct measurement of electric activity provides much more information by use of frequency analysis obtained by Fast Fourier Transformation (FFT), since single frequencies have been related to neurotransmitter activities. For example, slow delta waves seem to be under the control of acetylcholine [2]. Spectral power of theta waves changes in the presence of norepinephrine alpha2 receptor agonists [3]. The question now arises, if single frequencies at ROI's can be related not only to neurotransmitter activity but also to behavior. In order to find experimental evidence for involvement of different cortical regions during cognitive and emotional challenges, two techniques were combined, namely fast quantitative EEG analysis (Neurocode-Tracking) and Eye-Tracking [4]. The Eye-Tracking software served to present different cognitive and emotional challenges in form of tasks, pictures and video clips. Concomitant analysis of regional spectral power within six frequency ranges should not only give new insight into anatomical functionality with respect to cognition and emotion, but also provide information on particular neurotransmitters being involved. This becomes feasible by analysis of ultra-short recording epochs of 364 ms duration in comparison to time-averaged data on longer periods. These short periods of analysis have shown that the brain produces ultrashort explosive focal voltage peaks within one particular frequency range containing up to 10-fold amplitudes [5]. Therefore, it was hoped to get new insight into brain function with respect to neurotransmitters related to behavior.

\section{Method}

Sixty subjects aged 40 to 70 years were recruited within a clinical study aiming at the psychophysiological characterization of a food supplement during $7^{\text {th }}$ of September to $8^{\text {th }}$ of December 2015 by newspaper advertising. The study gained full approval from Ethics committee, Landesärztekammer Hessen, Im Vogelsgesang 3, D-60488 Frankfurt, Germany from the final protocol on 14.07.2015. All subjects gave full informed consent. Estimation of the number of subjects to be included into the study was performed by considering data from earlier experimental results obtained under a similar experimental design. Exclusion criteria were:

- Acute or chronic disease with an impact on the study, which becomes obvious by case history or clinical examination.

- Clinically relevant pathological findings from clinical and laboratory findings.

- Clinically relevant allergic symptoms.

- Detection of alcohol at the time of initial examination or on study day (positive alcohol test). Detection of drugs (positive drug test) at the time of initial examination.

- Consumption of clinically relevant medication during last fourteen days before and during the active study period based on the notification of the subject or his case history.

- Consumption of medication with primarily central action (i.e. psychotropic drugs or centrally acting antihypertensives). Known intolerance/hypersensitivity (allergy) to plant derived extracts or any of the ingredients of the investigational product (anamnestic). Presence of a rare, genetic disease such as fructose intolerance, glucose-galactose malabsorption or sucrase-isomaltase deficiency (anamnestic).

- $\quad$ BMI (Body Mass Index) $<18$ or $>32$.

- Consumption of unusual quantities or misuse of coffee (more than 4 cups a day), tea (more than 4 cups a day) or tobacco (more than 20 cigarettes per day).

- Participation in another clinical trial within the last 60 days.

- Positive pregnancy test (day A). 
- Lactation.

Only baseline recordings were processed in this paper in order to analyze the anatomical functionality of the electric responses induced by different cognitive and emotional challenges. Full data sets, taken before the intake of the preparation, were available for 57 subjects. Subjects underwent EEG recording during presentation of a video containing several cognitive tasks (fixation of a cross on the screen, picture comparison, Stroop test [6], memory test, arithmetic calculation, brain-teaser) as well as emotional challenges (three emotional pictures, a boring animal video, an exciting animal video and part of a horror video). Sequence of recordings is given in Table 1.

Quantitative EEG recording was performed as published [5]. In short, 17 electrode positions according to the so-called 10/20 system [7] were recorded from, and signals were assessed by Fast Fourier Transformation (FFT). Spectral power was calculated and processed further to give current source density [8]. These values are obtained by calculation of the second derivative after FFT. The software is commercially available from MEWICON CATEEM-Tec GmbH, Schwarzenberg am Böhmerwald, Austria. Firstly, data are documented as single representative epochs of 364 ms recording (bar graph and map (a)), directly cut out of the synchronized EnkephaloVision video. Median power is calculated by summing up the spectral power from all electrode positions and division by 17 (recordings are from 17 electrodes!). This value is set to 100\% and reflects local anatomical distribution of spectral power. Secondly, time-averaged data (difference to data recorded for 1 minute during the relaxed state) are calculated on each of the single scenes to give the median spectral power over that time period of recording from each electrode position as bar graph (b). Electric maps of averaged data (difference to data recorded during the relaxed state) were constructed after transforming power values into spectral colors and producing an additive color mixture in analogy to the RGB mode used in television providing a true experimental result, not a so-called "false color coding”. Mathematically, the maps represent a 64 channel EEG due to non-linear interpolation according to LaGrange (c). Statistical evaluation was performed by comparing the spectral power during presentation of the challenges to spectral power during the recording condition "eyes open during relaxation”. The non-parametric Sign Test, documented as statistical map for each brain region and for each frequency range in separate, was used (d). The bigger the block size, the larger the statistical significance compared to the recording condition "eyes open” during relaxation. An overview on the technology used has been published earlier [4] [9].

Table 1. Time line of cognitive and emotional challenges after the gong for synchronization purposes and reference time of one minute in a relaxed state.

\begin{tabular}{|c|c|c|}
\hline \multicolumn{3}{|c|}{$\begin{array}{c}\text { EnkephaloVision }=\text { Neurocode-Tracking in Combination with Eye-Tracking } \\
\text { Sequence of Audio-visual Presentations }\end{array}$} \\
\hline & Elements & Time \\
\hline 1 & Instructions of volunteers in the Neurocode-Tracking method & 5 min. $00 \mathrm{~s}$ \\
\hline 2 & Gong (for synchronization Neurocode-Tracking (qEEG and Eye-Tracking) & $21 \mathrm{~s}$ \\
\hline 3 & Black screen (eyes open) & 1 min. $00 \mathrm{~s}$ \\
\hline 4 & Fix cross 1 min (eyes open) & 1 min. $00 \mathrm{~s}$ \\
\hline 5 & Picture comparison ( $\mathrm{n}=1$ ) with 8 errors including instructions. & 1 min. $15 \mathrm{~s}$ \\
\hline 6 & Stroop test $(\mathrm{n}=8$ foils) including instructions. & 1 min. $00 \mathrm{~s}$ \\
\hline 7 & Memory test ( $\mathrm{n}=4$ tasks) including instructions. & 1 min. $35 \mathrm{~s}$ \\
\hline 8 & CPT-Test (concentration performance test) $(n=4)$ including instructions & 2 min. $20 \mathrm{~s}$ \\
\hline 9 & Brain-Teaser $n=10$ including instructions. & 5 min. $15 \mathrm{~s}$ \\
\hline 10 & Emotional Pictures $n=3$ & 1 min. $00 \mathrm{~s}$ \\
\hline 11 & Video “Animals” boring & $52 \mathrm{~s}$ \\
\hline 12 & Video "Horror" & 1 min. $11 \mathrm{~s}$ \\
\hline \multirow[t]{2}{*}{13} & Video “Animals” exciting & 1 min. $10 \mathrm{~s}$ \\
\hline & Total time excl. instructions of volunteers & $17 \mathrm{~min} .59 \mathrm{~s}$ \\
\hline
\end{tabular}




\section{Results}

The results of the present investigation are documented in two different ways reflecting two different length of recorded time periods. The first one shows representative recording epochs of $364 \mathrm{~ms}$. The second one documents time-averaged recording periods of different lengths (one scene). Both are shown within one image in order to compare single scenes with averaged data. Single scenes are cut out of a synchronized video clip containing the eye track results in combination with the quantitative EEG data (Neurocode-Tracking) called "EnkephaloVision.

\subsection{Analysis of Short Recording Epochs of $364 \mathrm{~ms}$ Duration}

The first processing of the data is documented as a bar graph and a map reflecting the so-called focal spectral median values (s. methods) as calculated over all cortical regions represented by 17 electrode positions according to the 10/20 system. Representative scenes are cut out of the synchronized EnkephaloVision videos as depicted in all figures under the heading of "a” representing the actual challenge with respect to the eye tracking result as red spot on the screen and the calculated spectral median power as bar graph and as map. The documented pattern of frequency changes was observed repeatedly during a particular scene. Please note the different scale on the ordinate of the bar graph in comparison to the averaged data! A short review of the last seconds of the time line is given for all frequencies on the lower right bottom for electrode position $\mathrm{F}_{7}$. For example, concentration on a fixed cross on the screen served as control and is documented in Figure 1(a). No difference is observed except for some increases of central alpha power (green within the map) during this moment of recording (364 ms).

A completely different result emerges when subjects compared two images searching for differences (picture comparison) as documented for a representative scene in Figure 2(a). Massive increases of delta and theta power at electrode positions $F_{7}$ and $F_{8}$ are observed during a representative scene matching the observations seen later within the averaged data. Within the time line reflecting seconds before this scene a large increase of theta power has occurred at electrode positions $\mathrm{F}_{7}$.

Next challenge consisted in the performance of the Stroop test. A representative scene taken during reading of the instruction revealed enormous increases of delta and theta power at the frontal electrodes $F_{7}$ and $F_{8}$ exceeding $7000 \%$ and nearly $4000 \%$, respectively, with respect to median spectral power (for definition see under methods). There were also median power increases at electrode position $T_{3}$ at this moment when looking at the word "Farbe". Details are given in Figure 3(a).

Increase of spectral power at lateral frontal areas represented by electrode positions $\mathrm{F}_{7}$ and $\mathrm{F}_{8}$ were also observed during performance of the arithmetic calculation test "CPT" reaching more than 2000\% spectral median power in combination with a considerable increase of beta1 power at electrode position $\mathrm{F}_{7}$. In addition, also the central region at $\mathrm{C}_{\mathrm{z}}$ showed increases. Largest increase happened to emerge during this scene at position $\mathrm{F}_{8}$ with respect to theta power in Figure 4(a).

The challenge of learning a sequence of 8 digits during performance of the memory test resulted in enormous increases of median spectral delta and theta power (more than 5000\%) in combination with a considerable increase of beta1 power at electrode position $\mathrm{F}_{7}$. Increase of beta1 power also emerged at position $\mathrm{F}_{3}$. Details are given in Figure 5(a).

During presentation of brain-teasers again massive increases of lateral frontal delta and theta power were observed in the range of $4000 \%-5000 \%$. On the left hemisphere at $\mathrm{F}_{8}$ also beta1 spectral power had increased. Massive beta power was also visible at this moment at electrode position $\mathrm{T}_{3}$ in Figure 6(a).

Presentation of an emotional picture only induced minor increases of spectral delta and theta power in the lateral frontal brain reaching about $1600 \%$ at the left side but considerably more on the right side, where also beta power increases was observed. Massive beta power increase was seen also at electrode position $\mathrm{T}_{3}$. Details are given in Figure 7(a).

During watching a boring animal video, a single scene revealed nearly no increases of delta and theta power at the left frontal electrode position but massive increases on the right hemisphere at position $\mathrm{F}_{8}$, where also alpha1, less alpha2 but a very large beta1 increase had emerged. Again beta spectral median power had increased at electrode position $\mathrm{T}_{3}$. Details are given in Figure $8(\mathrm{a})$.

During watching part of a horror video clip tremendous increases of frontal delta and theta power were observed during a single scene at electrode position $\mathrm{F}_{7}$. Even larger increases are seen at $\mathrm{F}_{8}$, where also alpha1, 


\section{Fix Cross / Eyes Open}

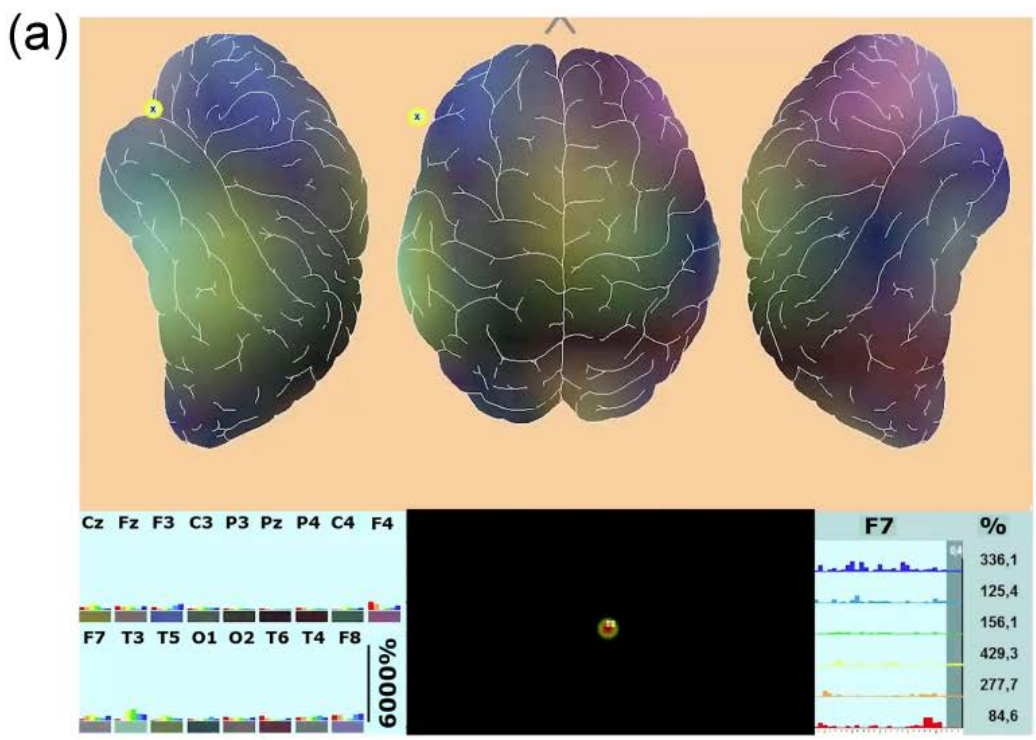

(b)

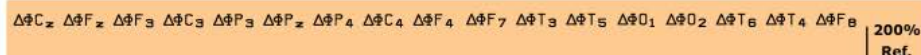

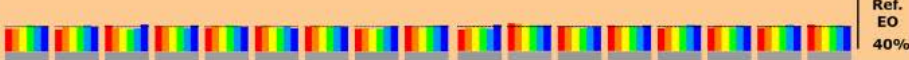

(c)
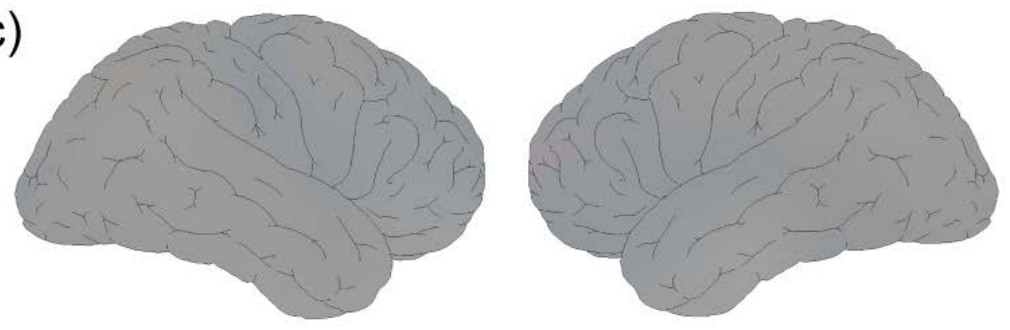

(d)
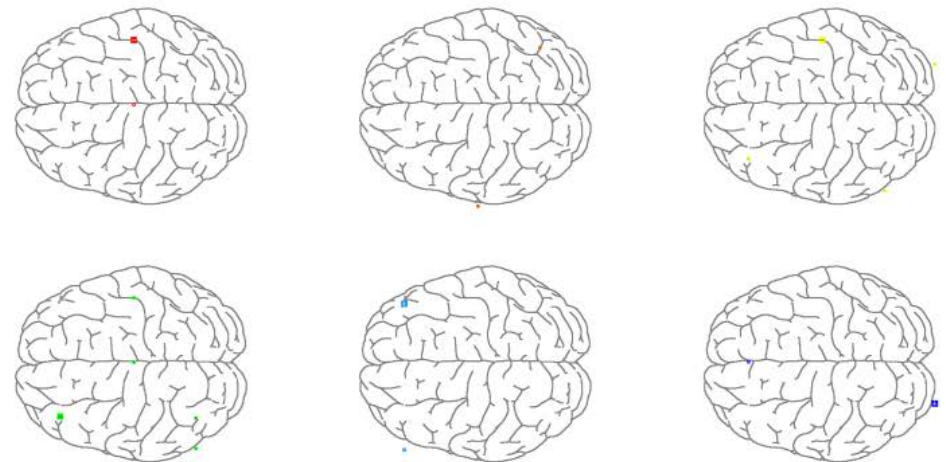

$\cdot p<0.20 \cdot p<0.10=p<0.05=p<0.01 \square p<0.005 \square p<0.001$

Figure 1. Spectral power changes during concentration on a "fix cross" in comparison to the relaxed state. (a) Representative single epoch of $364 \mathrm{~ms}$ showing electric map in the upper part and "fix cross" presentation in the lower middle part. Left lower side bar graph depicts spectral power difference to relaxation with ordinate up to $6000 \%$ with respect to median power (s. methods). Right lower side: Time line of spectral power for electrode position $\mathrm{F}_{7}$ in $\%$ of median power; (b) Spectral power changes in comparison to relaxation for all electrode positions; (c) Electric map showing difference to relaxation; (d) Statistical comparison to data recorded during relaxation. Please note different scaling on ordinate in (a) and (b). 


\section{Picture Comparison / Eyes Open}

(a)

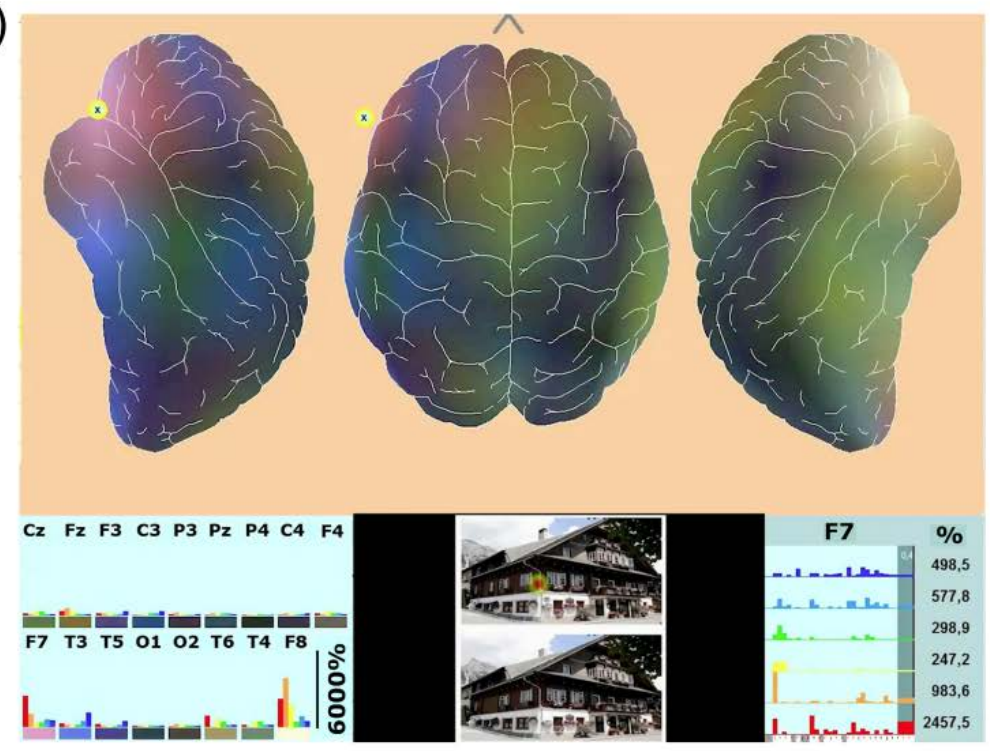

(b)

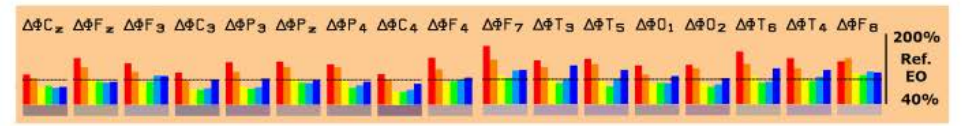

(c)

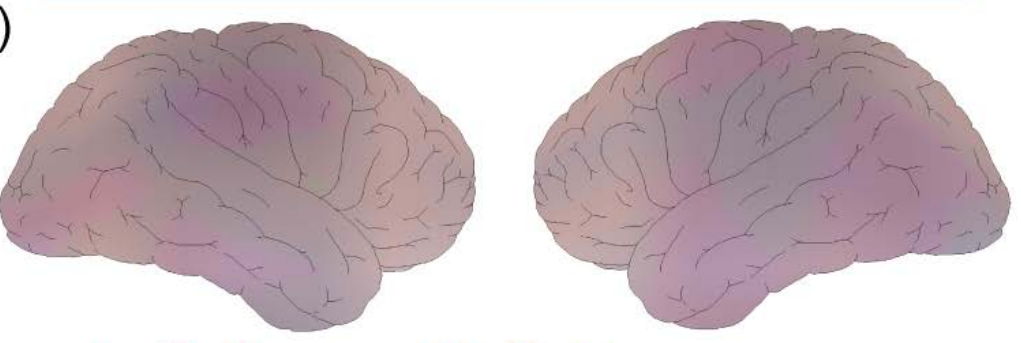

(d)
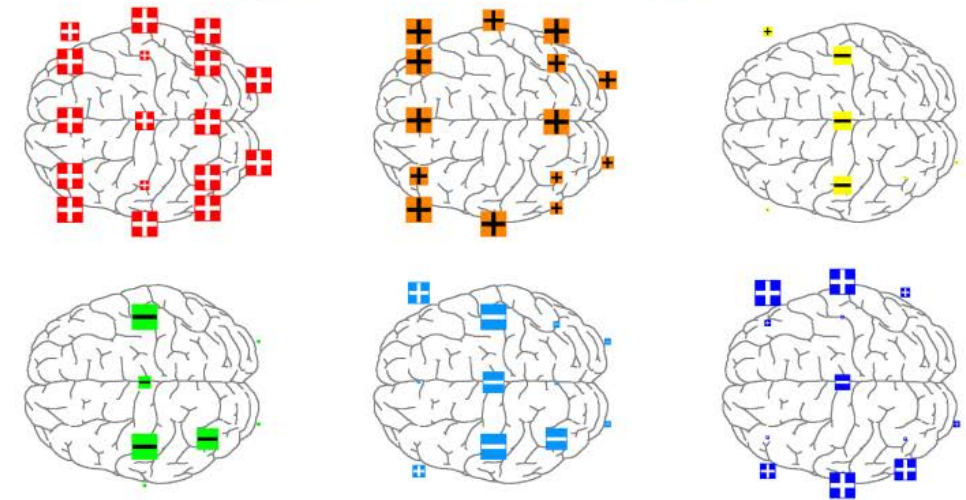

$\cdot p<0.20 \cdot p<0.10=p<0.05 \square p<0.01 \square p<0.005 \square p<0.001$

Figure 2. Spectral power changes during picture comparison in comparison to the relaxed state. (a) Representative single epoch of $364 \mathrm{~ms}$ showing electric map in the upper part and fix cross presentation in the lower middle part. Left lower side bar graph depicts spectral power difference to relaxation with ordinate up to $6000 \%$ with respect to median power (s. methods). Right lower side: Time line of spectral power for electrode position $\mathrm{F}_{7}$ in \% of median power; (b) Spectral power changes in comparison to relaxation for all electrode positions; (c) Electric map showing difference to relaxation; (d) Statistical comparison to data recorded during relaxation. Please note different scaling on ordinate in (a) and (b). 


\section{Stroop-Test / Eyes Open}

(a)

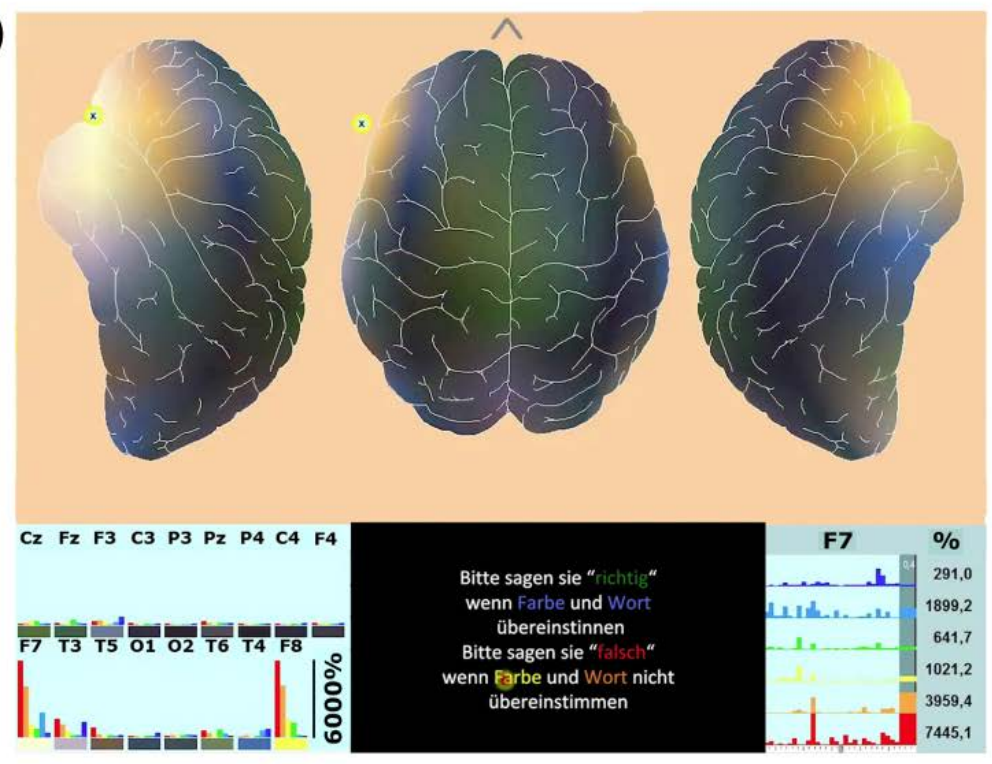

(b)

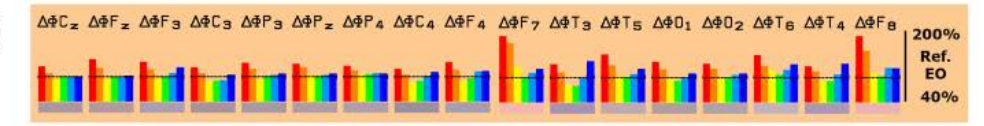

(c)
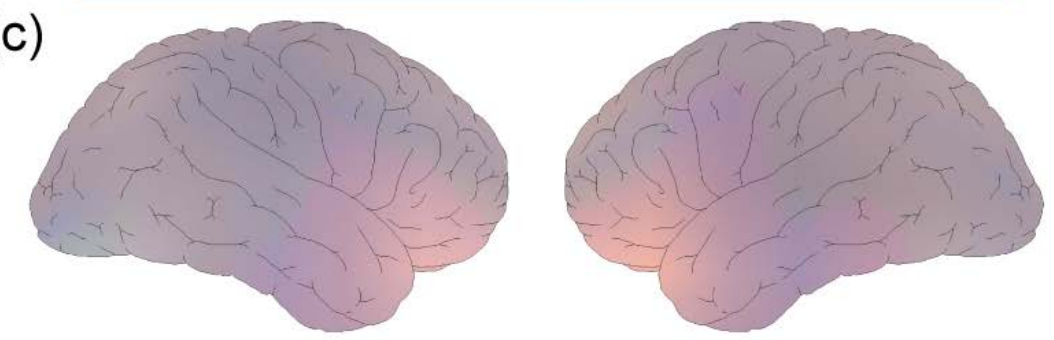

(d)
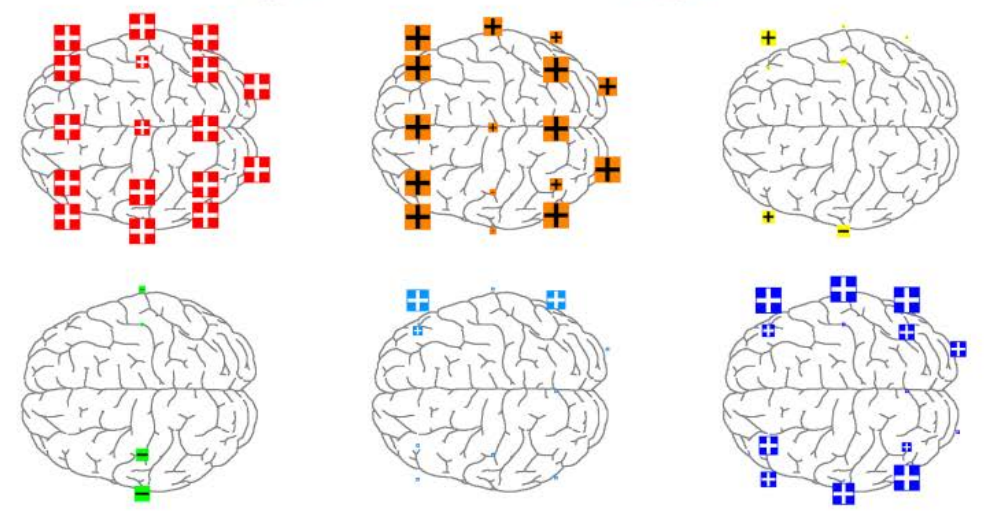

$\cdot p<0.20 \cdot p<0.10=p<0.05 \square p<0.01 \boxminus p<0.005 \square p<0.001$

Figure 3. Spectral power changes during performance of the Stroop test in comparison to the relaxed state. (a) Representative single epoch of 364 ms showing electric map in the upper part and fix cross presentation in the lower middle part. Left lower side bar graph depicts spectral power difference to relaxation with ordinate up to $6000 \%$ with respect to median power (s. methods). Right lower side: Time line of spectral power for electrode position $\mathrm{F}_{7}$ in \% of median power; (b) Spectral power changes in comparison to relaxation for all electrode positions; (c) Electric map showing difference to relaxation; (d) Statistical comparison to data recorded during relaxation. Please note different scaling on ordinate in (a) and (b). 


\section{CPT-Test / Eyes Open}

(a)

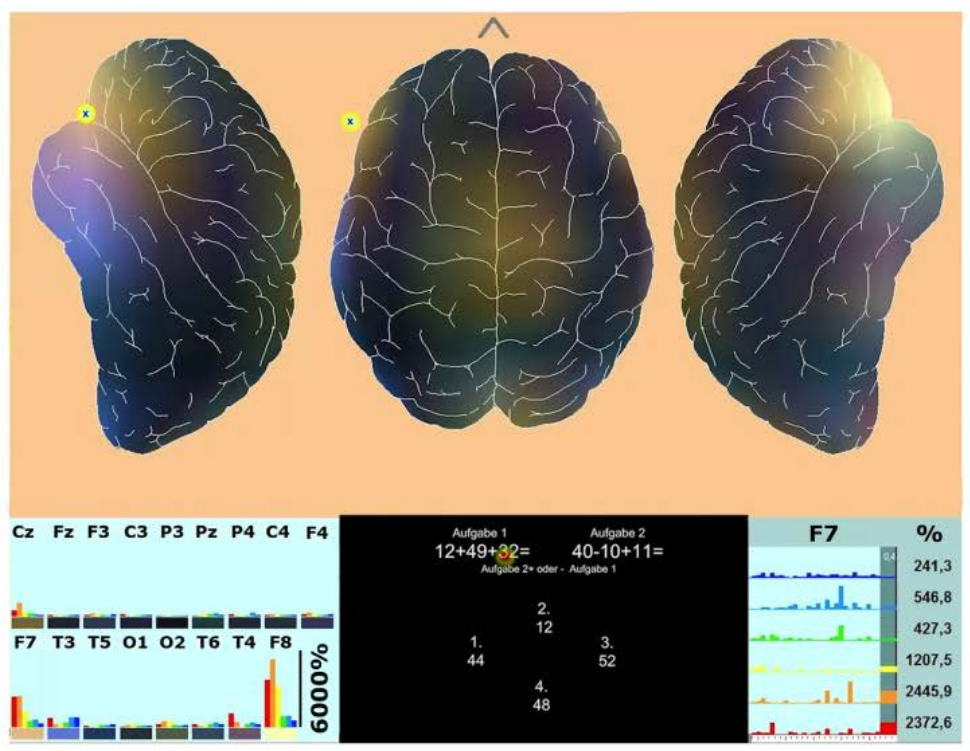

(b)

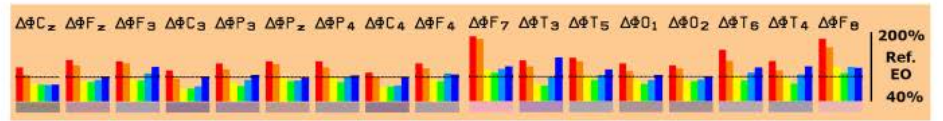

(c)
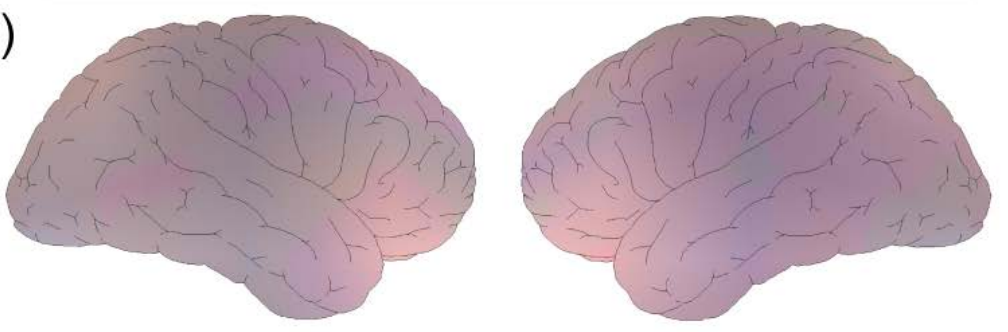

(d)
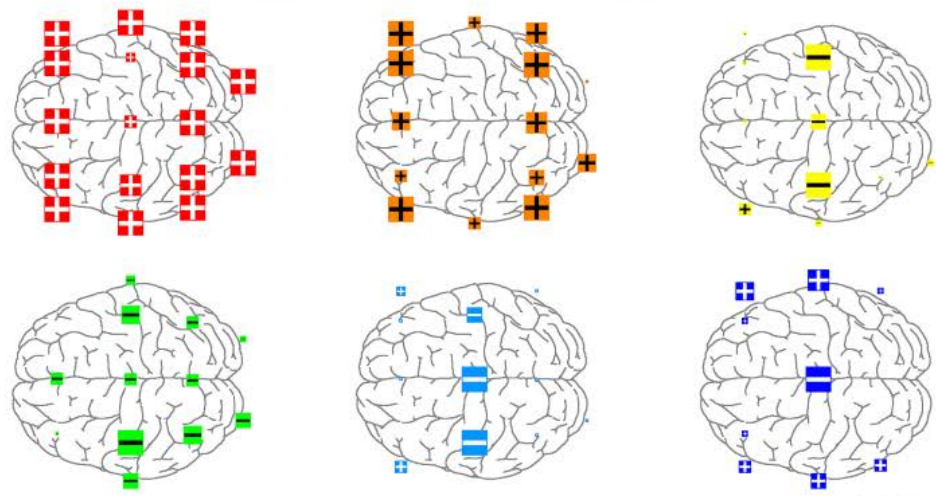

$\cdot \mathrm{p}<0.20 \cdot \mathrm{p}<0.10=\mathrm{p}<0.05 \mathrm{q}<0.01 \square \mathrm{p}<0.005 \square \mathrm{p}<0.001$

Figure 4. Spectral power changes during performance of the arithmetic concentration test in comparison to the relaxed state. (a) Representative single epoch of 364 ms showing electric map in the upper part and fix cross presentation in the lower middle part. Left lower side bar graph depicts spectral power difference to relaxation with ordinate up to $6000 \%$ with respect to median power (s. methods). Right lower side: Time line of spectral power for electrode position $\mathrm{F}_{7}$ in $\%$ of median power; (b) Spectral power changes in comparison to relaxation for all electrode positions; (c) Electric map showing difference to relaxation; (d) Statistical comparison to data recorded during relaxation. Please note different scaling on ordinate in (a) and (b). 


\section{Memory-Test / Eyes Open}

(a)

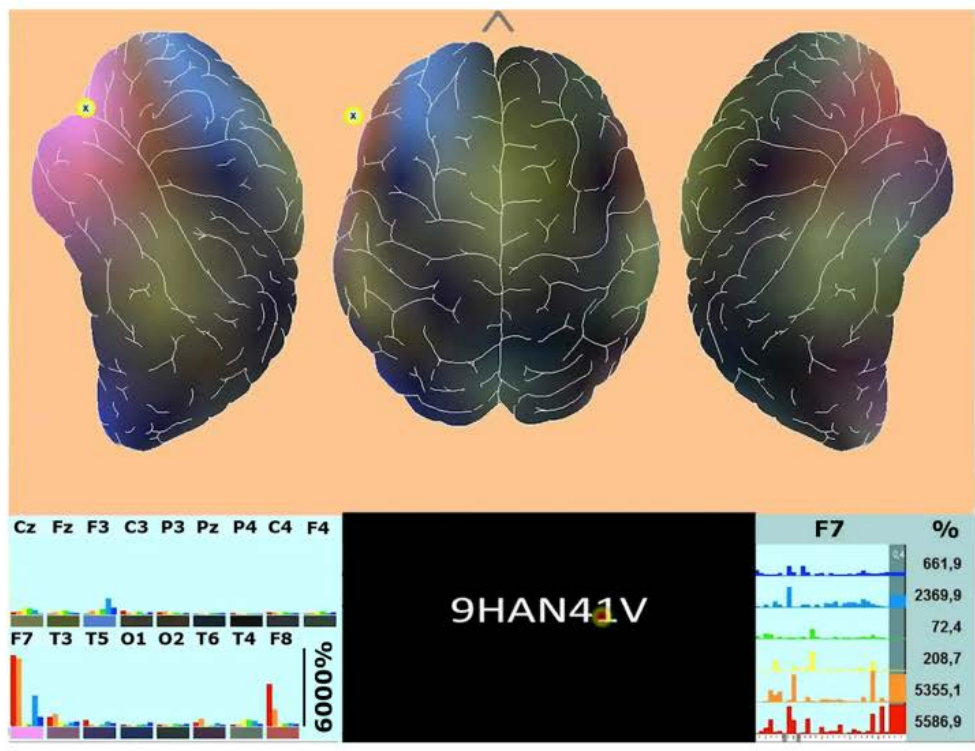

(b)

$\Delta \Phi C_{2} \Delta \Phi F_{2} \Delta \Phi F_{3} \Delta \Phi C_{3} \Delta \Phi P_{3} \Delta \Phi P_{2} \Delta \Phi P_{4} \Delta \Phi C_{4} \Delta \Phi F_{4} \quad \Delta \Phi F_{7} \Delta \Phi T_{3} \Delta \Phi T_{5} \Delta \Phi 0_{1} \Delta \Phi O_{2} \Delta \Phi T_{8} \Delta \Phi T_{4} \Delta \Phi F_{3}{ }_{200 \%}$

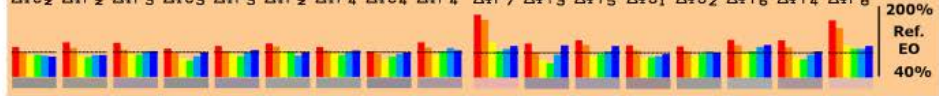

(c)
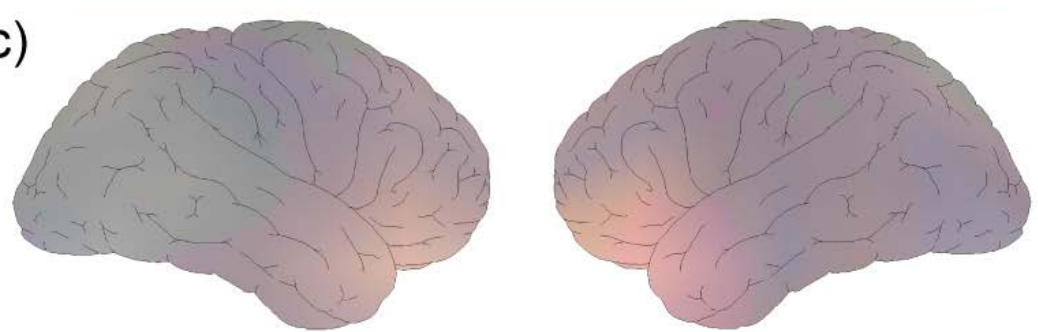

(d)
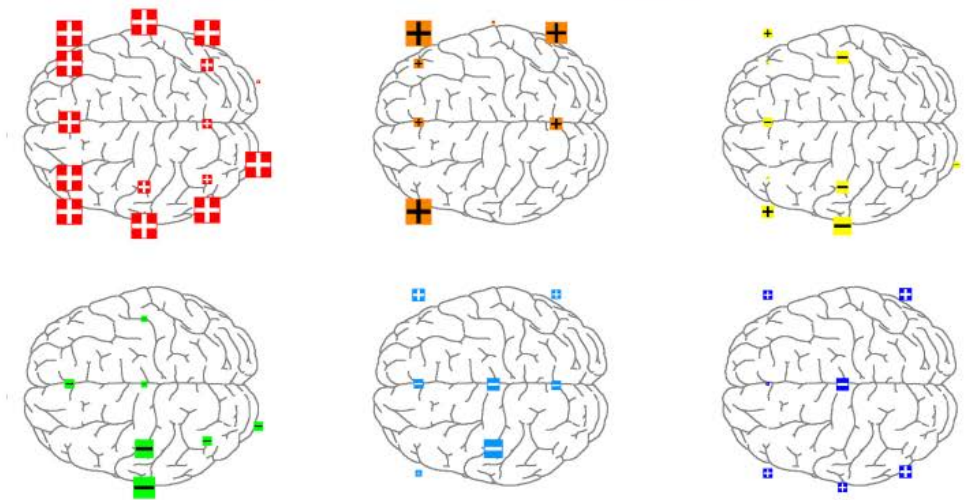

$\cdot p<0.20 \cdot p<0.10=p<0.05 \square p<0.01 \square p<0.005 \square p<0.001$

Figure 5. Spectral power changes during performance of the memory test in comparison to the relaxed state. (a) Representative single epoch of 364 ms showing electric map in the upper part and fix cross presentation in the lower middle part. Left lower side bar graph depicts spectral power difference to relaxation with ordinate up to $6000 \%$ with respect to median power (s. methods). Right lower side: Time line of spectral power for electrode position $\mathrm{F}_{7}$ in \% of median power; (b) Spectral power changes in comparison to relaxation for all electrode positions; (c) Electric map showing difference to relaxation; (d) Statistical comparison to data recorded during relaxation. Please note different scaling on ordinate in (a) and (b). 


\section{Brain-teaser / Eyes Open}

(a)

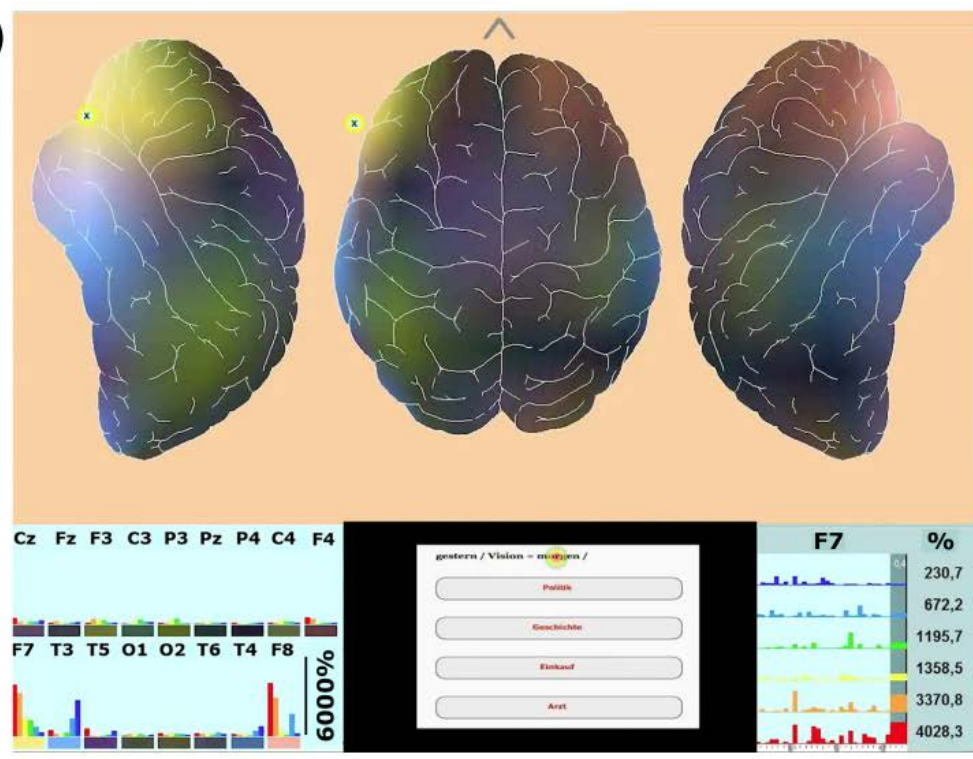

(b)

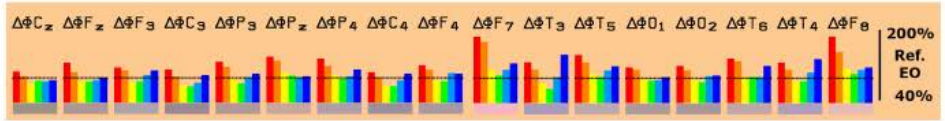

(c)
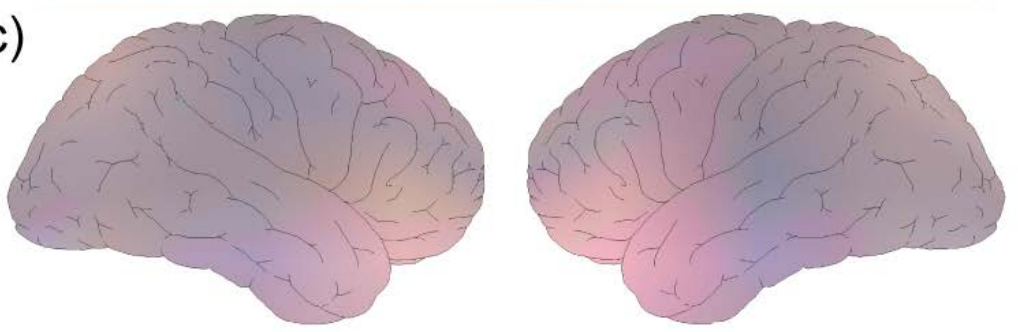

(d)
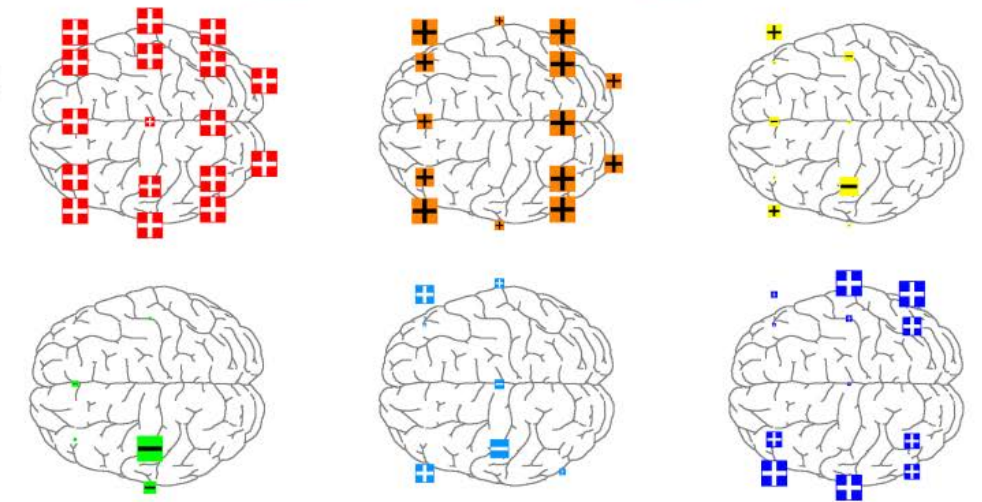

$\cdot p<0.20 \cdot p<0.10=p<0.05 \square p<0.01 \boxminus p<0.005 \square p<0.001$

Figure 6. Spectral power changes during presentation of brain-teasers in comparison to the relaxed state. (a) Representative single epoch of 364 ms showing electric map in the upper part and fix cross presentation in the lower middle part. Left lower side bar graph depicts spectral power difference to relaxation with ordinate up to $6000 \%$ with respect to median power (s. methods). Right lower side: Time line of spectral power for electrode position $\mathrm{F}_{7}$ in \% of median power; (b) Spectral power changes in comparison to relaxation for all electrode positions; (c) Electric map showing difference to relaxation; (d) Statistical comparison to data recorded during relaxation. Please note different scaling on ordinate in (a) and (b). 


\section{Emotional Pictures / Eyes Open}

(a)

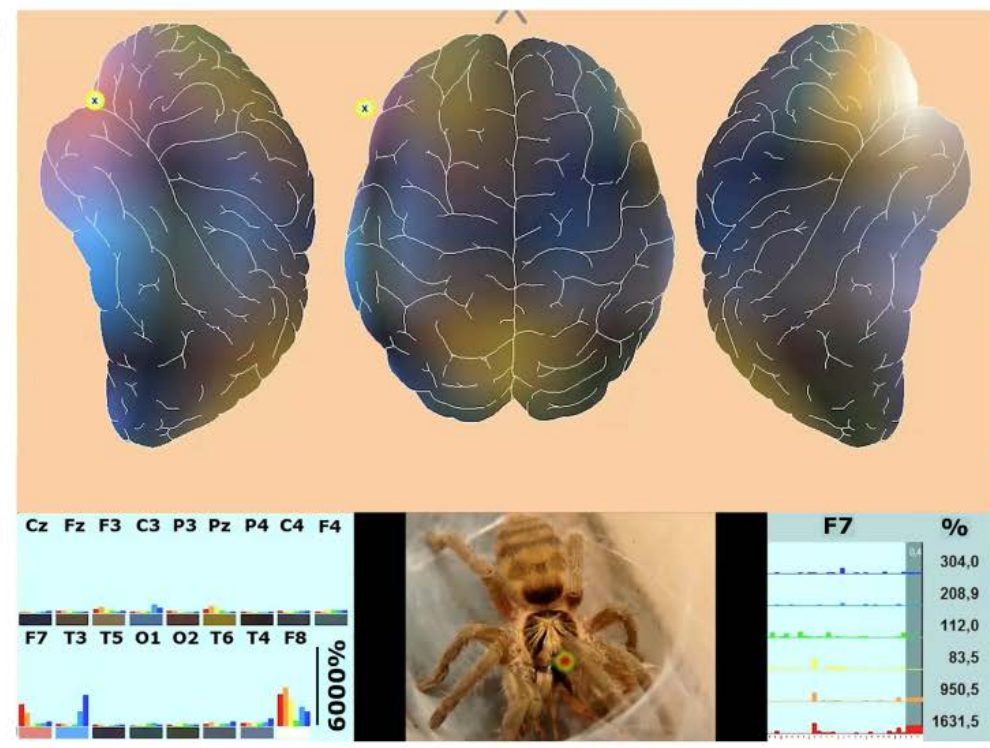

(b)

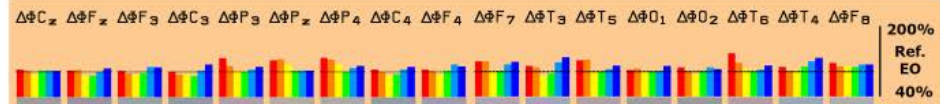

(c)
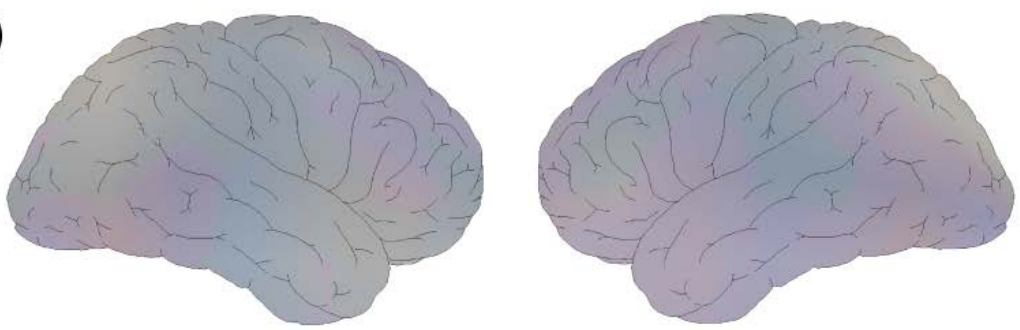

(d)
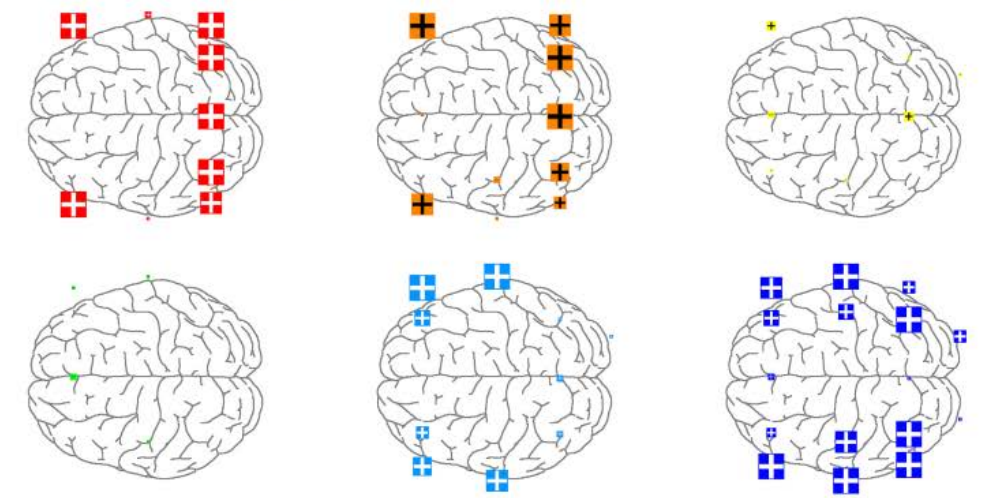

$\cdot p<0.20 \cdot p<0.10=p<0.05 \square p<0.01 \square p<0.005 \square p<0.001$

Figure 7. Spectral power changes during presentation of 3 emotional pictures in comparison to the relaxed state. (a) Representative single epoch of 364 ms showing electric map in the upper part and fix cross presentation in the lower middle part. Left lower side bar graph depicts spectral power difference to relaxation with ordinate up to $6000 \%$ with respect to median power (s. methods). Right lower side: Time line of spectral power for electrode position $\mathrm{F}_{7}$ in \% of median power; (b) Spectral power changes in comparison to relaxation for all electrode positions; (c) Electric map showing difference to relaxation; (d) Statistical comparison to data recorded during relaxation. Please note different scaling on ordinate in (a) and (b). 


\section{Animal Video Boring / Eyes Open}

(a)

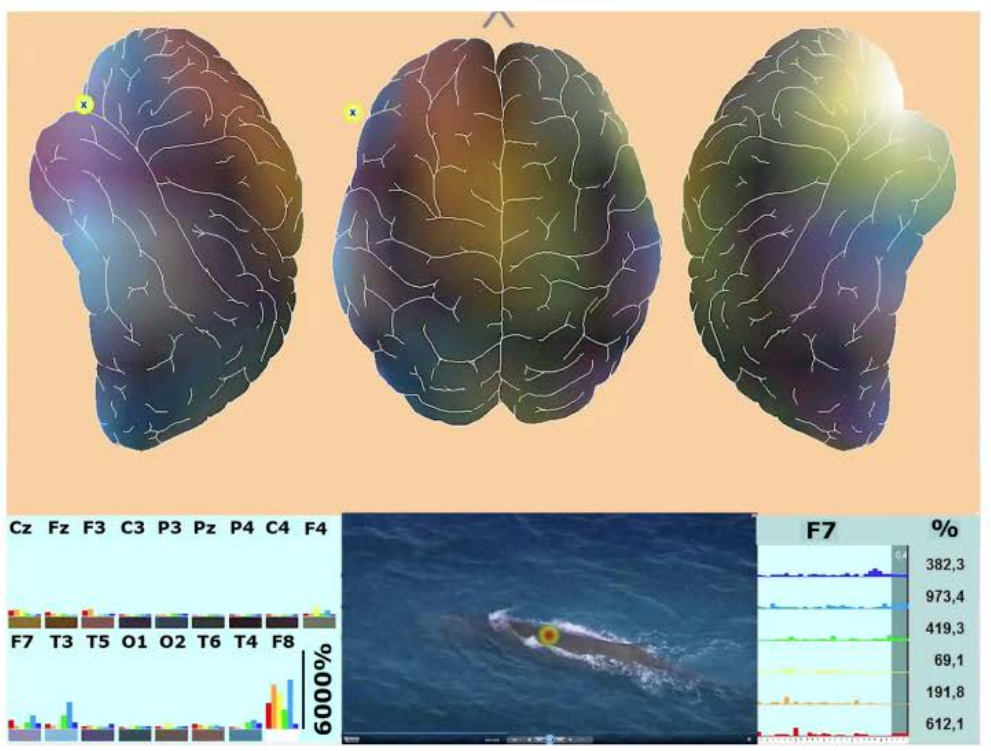

(b)

$\Delta \Phi C_{2} \Delta \Phi F_{2} \Delta \Phi F_{3} \Delta \Phi C_{3} \Delta \Phi P_{3} \Delta \Phi P_{2} \Delta \Phi P_{4} \Delta \Phi C_{4} \Delta \Phi F_{4} \Delta \Phi F_{7} \Delta \Phi T_{3} \Delta \Phi T_{5} \Delta \Phi 0_{1} \Delta \Phi O_{2} \Delta \Phi T_{3} \Delta \Phi T_{4} \Delta \Phi F_{3}{ }_{3}{ }^{200 \%}$

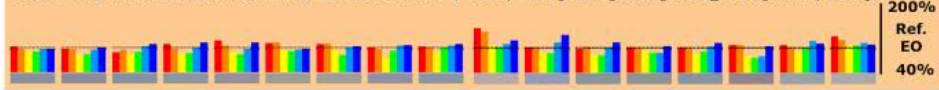

(c)
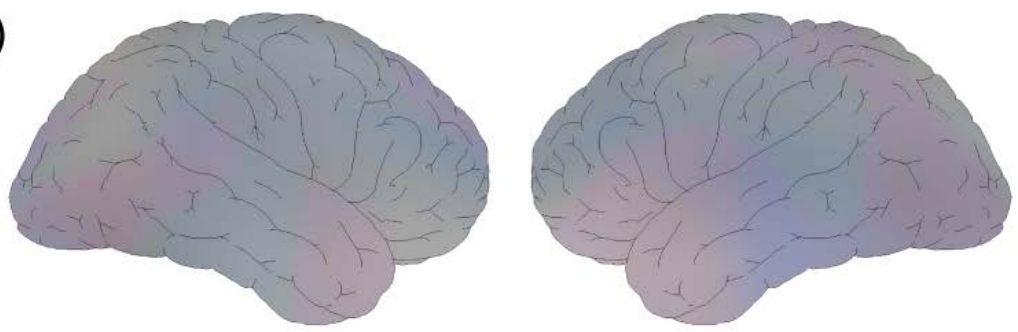

(d)
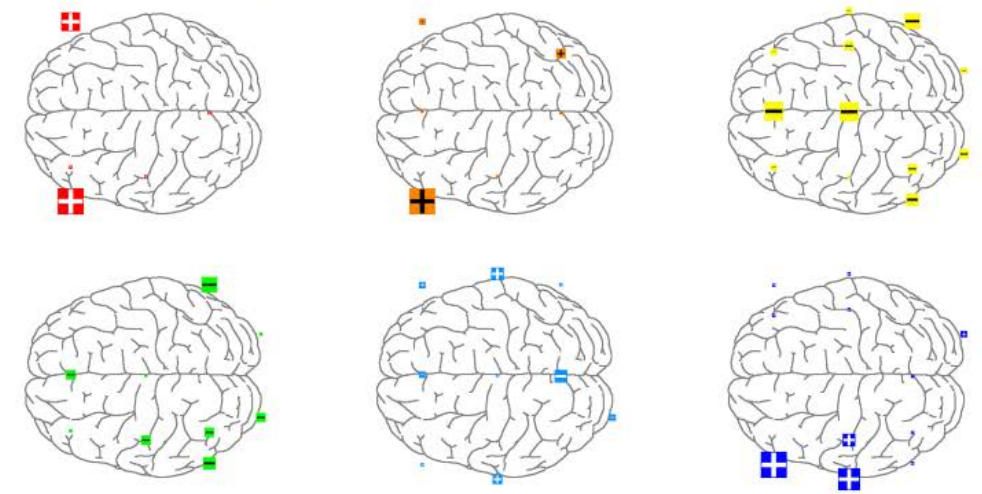

$\cdot p<0.20$ - $p<0.10=p<0.05=p<0.01 \square p<0.005 \square p<0.001$

Figure 8. Spectral power changes during watching a boring animal video in comparison to the relaxed state. (a) Representative single epoch of 364 ms showing electric map in the upper part and fix cross presentation in the lower middle part. Left lower side bar graph depicts spectral power difference to relaxation with ordinate up to $6000 \%$ with respect to median power (s. methods). Right lower side: Time line of spectral power for electrode position $\mathrm{F}_{7}$ in \% of median power; (b) Spectral power changes in comparison to relaxation for all electrode positions; (c) Electric map showing difference to relaxation; (d) Statistical comparison to data recorded during relaxation. Please note different scaling on ordinate in (a) and (b). 
beta1 and beta2 have increased in a massive manner. Thus, the right hemisphere was predominantly involved during that scene. Details are given in Figure 9(a).

During watching an exciting animal video, a single scene revealed increases of delta (about 1500\%) and more theta power (3300\%) at the left frontal electrode position, but tremendous increases on the right hemisphere at position $\mathrm{F}_{8}$, where spectral theta power had increased massively to more than $6000 \%$. In addition, spectral theta power had increased at $\mathrm{F}_{3}$ during this single scene. Details are given in Figure 10(a).

\subsection{Analysis of Time-Averaged Data with Respect to a Whole Challenge}

Before presentation of the challenges, one minute of EEG was recorded under the condition of a relaxed state (eyes open). Absolute spectral power within all six frequency ranges for every electrode position is documented in Table 2.

With respect to baseline absolute values during relaxation one can see, that there is higher spectral power in all frequency ranges at frontal electrode positions $\mathrm{F}_{7}$ and $\mathrm{F}_{8}$ as observed in all earlier studies. Second highest values are observed at positions $\mathrm{T}_{3}$ and $\mathrm{T}_{4}$. These data were set to $100 \%$ and served as reference. All data collected during the diverse cognitive and emotional challenges are given as difference to these reference values recorded in the presence of the relaxed state and documented within a bar graph "b" in all figures. Average maps calculated for each total challenge are documented under "c". Statistical comparison between challenges and these reference values in the relaxed state is calculated for all 102 parameters separately and documented as a statistic map. Depending on the type of challenge different patterns of electric activation were observed as shown in all figures under "d".

During concentration on a fixed cross in the middle of the screen no electric response is observed after calculation of differences to the relaxed state.

Opposite to this, recording during a cognitive challenge like comparing two images for detection of differences (Picture comparison) revealed strong and statistically significant increases of delta with respect to all brain regions and increases of theta spectral power except for the central area, whereas alpha power decreased in a statistically significant manner in the central region. With respect to beta1 power a frontal increase and a central decrease was observed. Beta2 power increased fronto-temporally (Figures 2(b)-(d)).

Another cognitive challenge, the Stroop test, also induced massive general increases in spectral delta and theta power with respect to all brain areas, but hardly decreases in alpha spectral power. Beta1 spectra power only increased in the right temporal lobe significantly. However, beta2 spectral power showed most statistically significant increases frontally and in the temporal lobe with some increases in the lateral parietal lobe besides increases in the occipital lobe. Data are documented in Figures 3(b)-(d).

A mentally very demanding cognitive challenge is the arithmetic concentration test "CPT". During performance of this test a general increase of delta spectral power is observed at all electrode positions as already seen in the image comparison and Stroop test. With respect to theta power central regions are spared from changes. However, frontal, temporal and parietal areas show a highly significant increase of theta power. Alpha1 spectral power decreases in central positions. Alpha2 spectral power decreases in general in a statistically significant manner, too. Beta1 spectral power increases frontally $\left(\mathrm{F}_{7}\right.$ and $\left.\mathrm{F}_{8}\right)$, but decreases centrally. Similar to beta1 power beta2 power increases fronto-temporally significantly, but decreases centrally (Figures 4(b)-(d)).

Performance of the memory test led to highly significant increases of spectral delta power in frontal and temporal regions. With respect to theta spectral power lateral frontal brain $\left(\mathrm{F}_{7}\right.$ and $\left.\mathrm{F}_{8}\right)$ and the parietal lobe showed highly significant increases. Within this region also alpha1 spectral power increased to some extent, whereas other central areas showed a decrease. Whereas alpha2 power decreased on the left hemisphere, beta1 power changes were similar to those observed with respect to alpha1. Beta2 spectral power increases fronto-temporally, but decreases centrally. Results are documented in Figures 5(b)-(d).

A very interesting cognitive challenge is obtained by presenting brain-teasers. This represents a rather high mental load. During working on and solving the questions a general increase of delta power is observed on the whole surface in a statistically significant manner. Theta power increased in the frontal, temporal and parietal area in comparison to relaxation. Alpha1 power increased in the lateral frontal region represented by electrode positions $\mathrm{F}_{7}$ and $\mathrm{F}_{8}$. Lateral frontal positions showed an increase of beta1 power, whereas in the central region beta1 power decreased. Beta2 power increases in the lateral frontal, temporal and parietal lobe in a highly significant manner. Results are depicted in Figures 6(b)-(d). 


\section{Horror Video / Eyes Open}

(a)

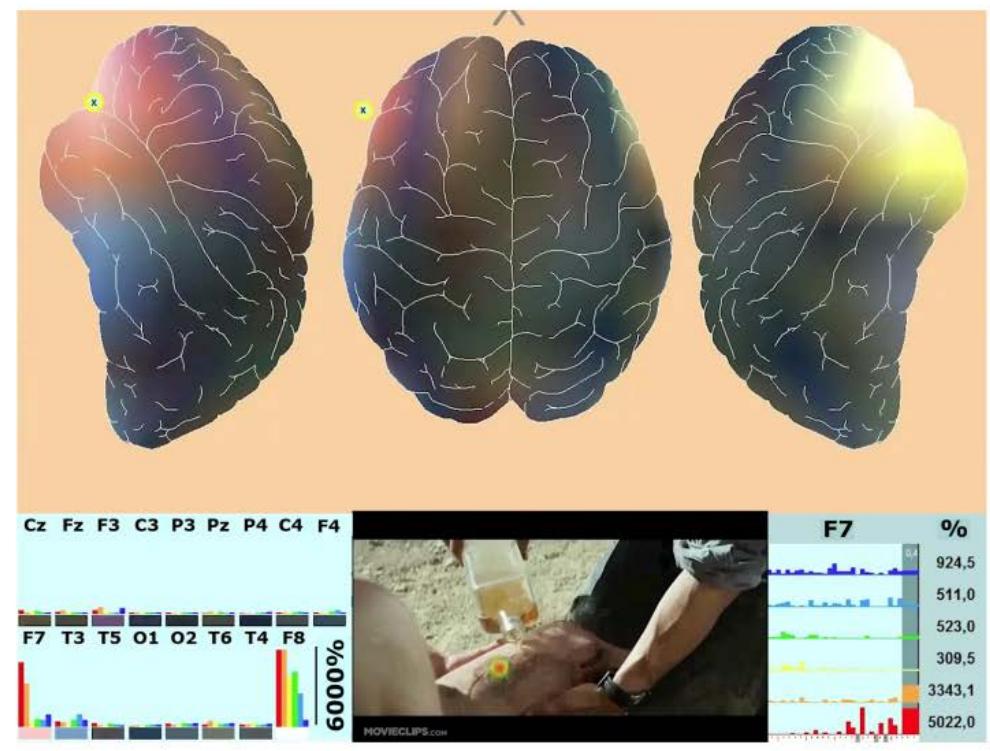

(b)

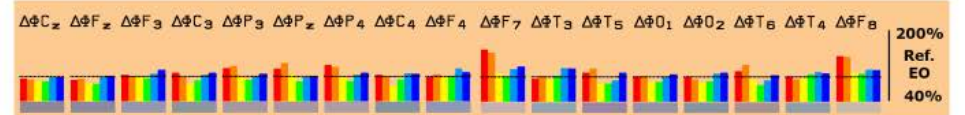

(c)
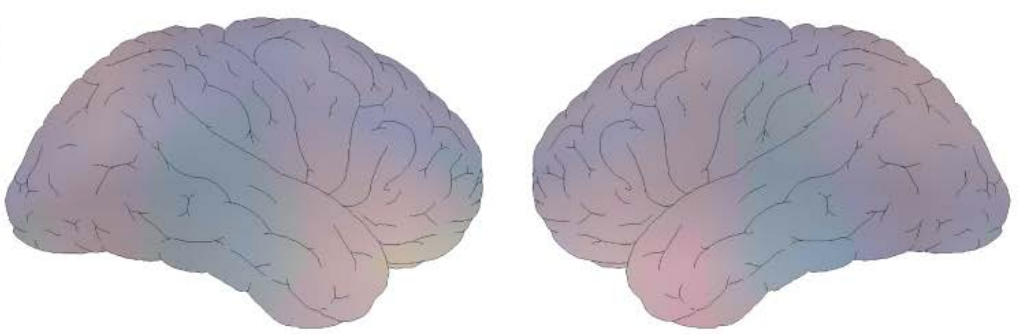

(d)
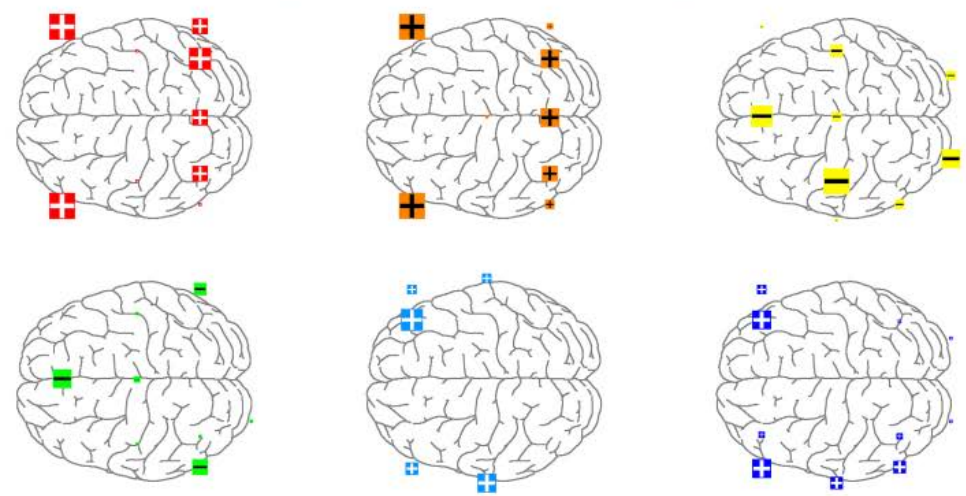

$\cdot p<0.20 \cdot p<0.10=p<0.05 \square p<0.01 \square p<0.005 \square p<0.001$

Figure 9. Spectral power changes during watching scenes from a horror video in comparison to the relaxed state. (a) Representative single epoch of 364 ms showing electric map in the upper part and fix cross presentation in the lower middle part. Left lower side bar graph depicts spectral power difference to relaxation with ordinate up to $6000 \%$ with respect to median power (s. methods). Right lower side: Time line of spectral power for electrode position $\mathrm{F}_{7}$ in \% of median power; (b) Spectral power changes in comparison to relaxation for all electrode positions; (c) Electric map showing difference to relaxation; (d) Statistical comparison to data recorded during relaxation. Please note different scaling on ordinate in (a) and (b). 


\section{Animal Video Exciting / Eyes open}

(a)

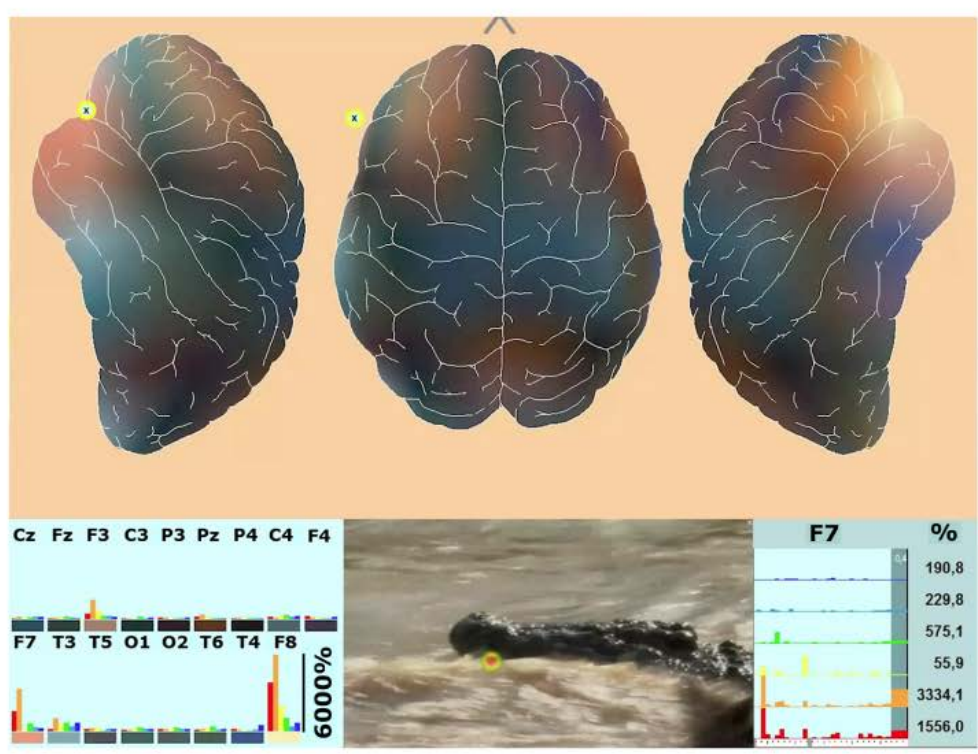

(b)

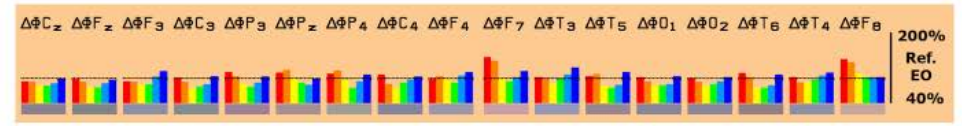

(c)

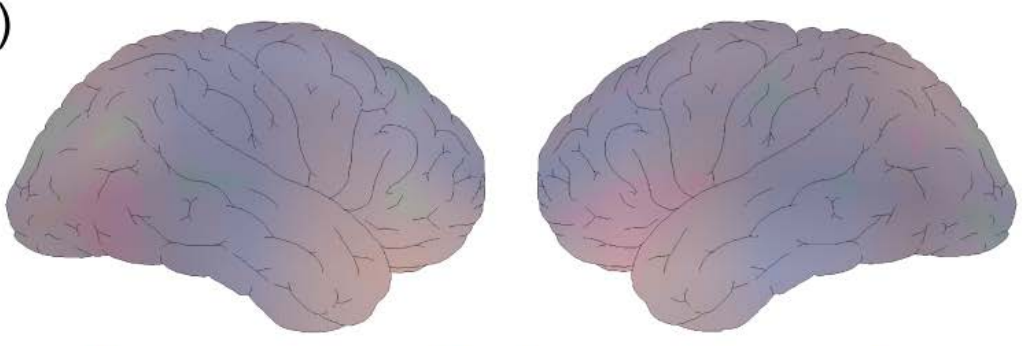

(d)
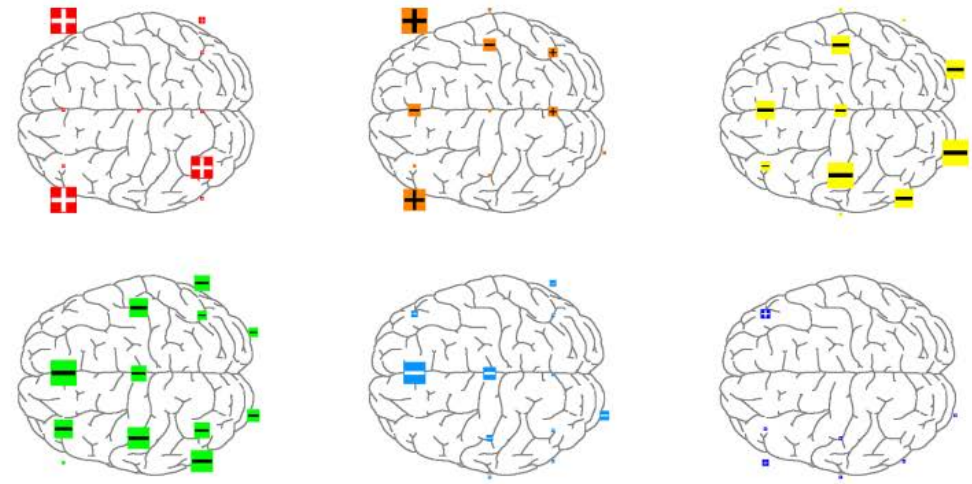

$\cdot p<0.20$ - $p<0.10=p<0.05 \square p<0.01 \square p<0.005 \square p<0.001$

Figure 10. Spectral power changes during watching an exciting animal video in comparison to the relaxed state. (a) Representative single epoch of 364 ms showing electric map in the upper part and fix cross presentation in the lower middle part. Left lower side bar graph depicts spectral power difference to relaxation with ordinate up to $6000 \%$ with respect to median power (s. methods). Right lower side: Time line of spectral power for electrode position $\mathrm{F}_{7}$ in \% of median power; (b) Spectral power changes in comparison to relaxation for all electrode positions; (c) Electric map showing difference to relaxation; (d) Statistical comparison to data recorded during relaxation. Please note different scaling on ordinate in (a) and (b). 
Table 2. Documentation of spectral power within each frequency range as $\mu \mathrm{V}^{2}$ for every electrode position. Med $=$ median calculated over all electrode positions. Electrode positions are given according to the so-called 10/20 system.

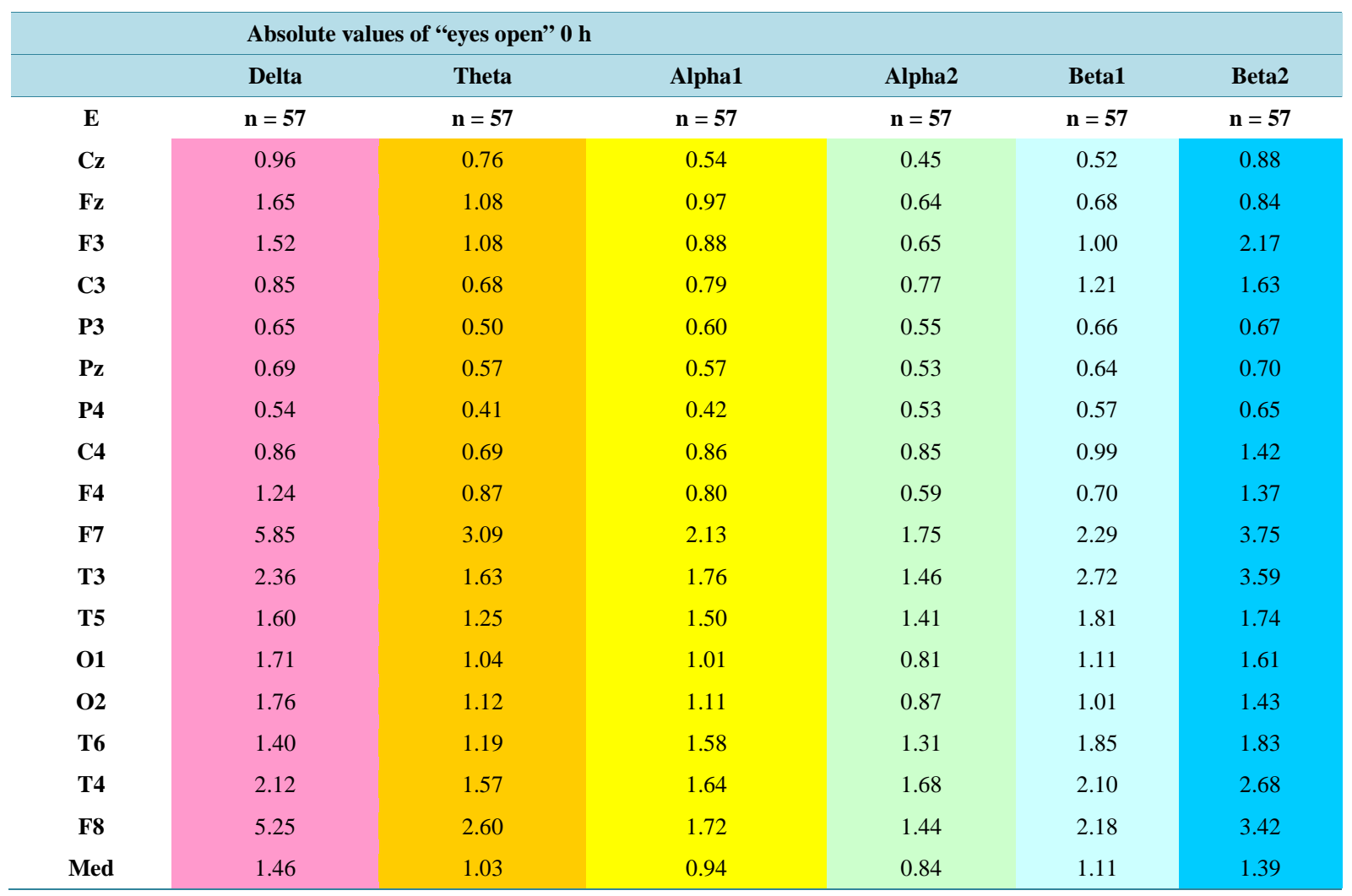

Besides cognitive challenges, four emotional challenges were also presented. The emotional part of the experiment started with the presentation of 3 emotional pictures. Under this condition the time-averaged data revealed a statistically highly significant increase of delta and theta spectral power at lateral frontal electrode positions $\left(\mathrm{F}_{7}\right.$ and $\left.\mathrm{F}_{8}\right)$ as well as all parietal electrodes and temporal position $\mathrm{T}_{5}$ and $\mathrm{T}_{6}$. Almost no changes were observed with respect to alpha activity. However, beta1 spectral power increased at all frontal positions except for $\mathrm{F}_{\mathrm{z}}$, but also at temporal position $\mathrm{T}_{3}$. With respect to beta2 spectral power all frontal positions showed statistically significant increased power. In addition, also central regions $C_{3}$ and $C_{4}$ as well as $P_{3}$ and $P_{4}$ likewise showed increase of power. Finally, also at temporal positions $\mathrm{T}_{3}$ and $\mathrm{T}_{4}$ increases of beta2 power were statistically significant (Figures 7(b)-(d)).

Watching a boring animal video led to selective statistically significant increases of lateral frontal delta and theta power, but a general decrease of spectral power in the alpha range. Beta1 power is hardly affected, but beta1 power increases in the fronto-temporal area within the left hemisphere (Figures 8(b)-(d)).

Watching scenes of a horror video induced increases of lateral frontal and parietal delta and theta spectral power in comparison to relaxation in a statistically significant manner. Opposite to this, alpha power decreases in general, leading in some cases to statistically significant differences to the relaxation state. Both beta ranges showed statistically significant increases in the frontal and temporal lobe (Figures 9(b)-(d)).

In comparison to the changes observed during the boring animal video, the exciting animal video induced some stronger effects, since now also the parietal lobe changed its activity in terms of producing increases of delta and theta activity in addition to the lateral frontal lobe. Also stronger general decreases of alpha activity were observed. Beta activity was only affected to a minor degree (Figures 10(b)-(d)).

\section{Discussion}

This investigation aimed at the detection of functional changes of electric activity with respect to 17 brain areas during various cognitive and emotional challenges as presented by eye tracking software. This approach not on- 
ly allowed for collecting average data on single challenges but also allowed for documentation of electric patterns during single scenes, where the position of the eyes was documented for a single epoch. Surprisingly, this kind of concomitant analysis revealed tremendous transient increases of focal spectral power (up to more than $6000 \%$ ), when calculated as \% of median power. This has not been known before, since such short periods of time have been analyzed by us for the first time and are restricted to the length of $364 \mathrm{~ms}$ [5]. This epoch length corresponds quite well to the processing time of the brain during presentation of audio-visual information as known from recording of the so-called P300, a wave emerging at about 300 ms after presentation of the stimulus [10].

Lateral frontal brain was involved more or less during all challenges. With respect to memory, for example, frontal and temporal lobe have been assigned as brain regions heavily involved [11]. Predominant changes were observed first line in the delta frequency range, where increases were observed during all scenes. This implicates, that cholinergic transmission is modulated throughout cognitive and emotional processing as for example documented in the right prefrontal lateral cortex within a single epoch together with theta and beta1 increase (Figure 8(a)). Interestingly, only occasionally other electrode positions were involved at the same time with respect to single frequencies like beta2 at electrode position $\mathrm{T}_{3}$ as can be seen from the averaged data. Other scenes showed different patterns. The ones shown were selected as representative to be compared to the averaged data. This result questions so-called coherence analysis on averaged data, since single epochs of activity show different combinations of frequency changes. The ultrashort epochs of 364 ms duration show very clearly tremendous transient increases of spectral power confined to local brain regions, very often only within one hemisphere. This questions the interpretation of time-averaged data with respect to local involvement of electric circuits during a cognitive or emotional reaction. Instead of measuring the so-called P300, serial analysis of the ultra-short single epochs of 364 ms especially in combination with eye tracking provides much more insight into electric active brain processes during cognitive or emotional challenges.

It is already well known, that there are hemispheric differences during mental processing. For example, with respect to retrieval of information from the episodic memory and from the knowledge domain with the first being associated with the right and the second with the left hemisphere [12] [13]. According to a PET study it was found that for encoding of episodic information the left prefrontal cortex was differentially activated, whereas for retrieval the right prefrontal cortex was activated [14]. Thus, the present analysis also showed the involvement of brain regions which had been previously recognized to play a role during cognitive processing.

Coming now to the averaged data. Even during one minute of relaxation a small mental activation takes place in the lateral frontal cortex as represented by the electrode positions $\mathrm{F}_{7}$ and $\mathrm{F}_{8}$. Therefore, data recorded during the cognitive and emotional challenges are documented by calculating the difference to the relaxed state with open eyes in order to be able to better differentiate specific patterns of the challenges from each other. As observed also in earlier studies using classic analysis of EEG fronto-temporal delta and theta spectral power increases during mental loads like the d2-concentration endurance test, an arithmetic concentration test and a memory test [15]. The present investigation confirms this result and shows that it depends on the type of challenge, how strong these increases of slow waves develop. According to the differences observed, it seems that the stronger the psychic demand, the higher the increase of fronto-temporal-parietal spectral power. The results clearly demonstrate that there is an anatomical functionality with respect to the involvement of the frontal cortex and temporal lobe, and also the parietal lobe, during cognitive and emotional challenges. Interestingly, predominant changes of spectral power are observed with respect to slow waves, which in the common textbooks are exclusively related to sleep. On the other hand, decreases of alpha power are observed mainly in central areas as already known from the literature [16]. Increases of frontal theta and central decreases of alpha spectral power have been related to cognitive processes on the base of averaged data [17]. These results were confirmed now. However, recording of the ultrafast transient spectral power peaks during cognitive and emotional processing revealed that emotional challenges likewise led to similar changes of frequency power as observed during cognitive challenges. This must be interpreted in a sense that the same electric circuits serve for both cognitive and emotional processing.

During some challenges increases of beta power are observed in the frontal as well as temporal lobe. The results are in favor of the involvement of many brain areas during mental processing. Surprisingly, there are only quantitative differences between cognitive and emotional challenges with respect to the involvement of several brain regions. Common to all challenges is an increase of delta power at the frontal electrodes $F_{7}$ and $F_{8}$. After deduction of the power values during the relaxed state, a decrease of delta power was never observed in any of 
the electrode positions. However, depending on the challenge more or less regions were involved. This increase of spectral delta power indicates a major involvement of the cholinergic transmitter system during processing of cognitive as well as emotional challenges not only confined to lateral prefrontal brain [2]. The same pattern was seen with respect to theta power, where also only increases were documented during the different challenges. Theta waves are under the control of norepinephrine. A different picture emerged with respect to alpha1 spectral power. An increase of power was only observed at the lateral frontal electrode positions. Alpha1 waves are related to serotonergic transmission [18]. Otherwise, always either no effect or an attenuation was seen with respect to all other electrode positions. No increases of alpha2 power have been detected at all. However, strongest general attenuation was observed during arithmetic calculation and during watching the exciting animal movie. Since this frequency range has been shown to reflect dopaminergic activity [19], this transmitter obviously plays a major role during processing of cognitive as well as emotional challenges. With regard to beta1 and beta2 spectral power, representing glutamatergic and GABA-ergic transmission, respectively, increases emerged more or less at frontal and temporal regions, whereas attenuation was seen centrally. Accordingly, strongest involvement of GABA-ergic transmission was observed during performance of the Stroop test, solving brain teasers and looking at emotional pictures.

\section{Conclusion}

In summary, the spectral power of frequencies from the different brain regions always reacted in the same direction during all challenges, if they responded at all. Depending on the type of challenge the frontal lobe responded first, followed by the parietal lobe and temporal lobe. Thus, particular neuronal circuits must exist, which connect these areas with each other and which respond to the mental load of the particular challenge or task to be performed. Obviously, in terms of functional anatomy local neuronal activation and information processing depends strongly on the type of task or challenge and may involve more or less several brain areas. The advantage of recording electric activity consists in taking a look at different frequencies representing neurotransmitter activity and several brain regions at the same time during many audio-visual presentations, including for ultrashort periods. The disadvantage might be seen in the fact that only cortical regions can be monitored. Finally, since quantitative EEG recording revealed anatomical functionality with respect to particular brain regions related to different challenges, drug induced changes can also be monitored in a predictive way.

\section{References}

[1] Hurley, R.S., Bonakdarpour, B., Wang, X. and Mesulam, M.M. (2015) Asymetric Connectivity between the Anterior Temporal Lobe and the Language Network. Journal of Cognitive Neuroscience, 27, 464-473. http://dx.doi.org/10.1162/jocn_a_00722

[2] Dimpfel, W. (2005) Pharmacological Modulation of Cholinergic Brain Activity and Its Reflection in Special EEG Frequency Ranges from Various Brain Areas in the Freely Moving Rat (Tele-Stereo-EEG). European Neuropsychopharmacology, 15, 673-682. http://dx.doi.org/10.1016/j.euroneuro.2005.03.006

[3] Dimpfel, W. and Schober, F. (2001) Norepinephrine, EEG Theta Waves and Sedation in the Rat. Behavioural Pharmacology, 1, 89-97.

[4] Dimpfel, W. (2015) Neuromarketing: Neurocode-Tracking in Combination with Eye-Tracking for Quantitative Objective Assessment of TV Commercials. Journal of Behavior and Brain Science, 5, 137-147. http://dx.doi.org/10.4236/jbbs.2015.54014

[5] Dimpfel, W. and Hofmann, H.C. (2014) Neurocode-Tracking Based on Quantitative Fast Dynamic EEG Recording in Combination with Eye Tracking. World Journal of Neuroscience, 4, 106-119. http://dx.doi.org/10.4236/wjns.2014.42013

[6] Stroop, J.R. (1935) Studies of Interferences in Serial Verbal Reactions. Journal of Experimental Psychology, 18, 643662. http://dx.doi.org/10.1037/h0054651

[7] Jasper, H.H. (1958) The Ten-Twenty Electrode System of the International Federation. Electroencephalography and Clinical Neurophysiology, 10, 371-375.

[8] Harmony, T., Fernandez-Bouzas, A., Marosi, E., Fernandez, T., Bernal, J., Rodriguez, M., Reyes, A., Silva, J. and Alonso, M.G. (1993) Correlation between Computed Tomography and Voltage and Current Source Density Spectral Parameters in Patient with Brain Lesions. Electroencephalography and Clinical Neurophysiology, 87, 196-205. http://dx.doi.org/10.1016/0013-4694(93)90019-R 
[9] Dimpfel, W. (2011) Enkephaloglyphen. Spektrale Signaturen der elektrischen Gehirntätigkeit als Spiegel der Psyche. Books on Demand GmbH, Norderstetten.

[10] Polich, J. (2004) Clinical Application of the P300 Event-Related Brain Potential. Physical Medicine and Rehabilitation Clinics of North America, 15, 133-161. http://dx.doi.org/10.1016/S1047-9651(03)00109-8

[11] Kroll, N.E.A., Markowitsch, H.J., Knight, R.T. and von Cramon, D.Y. (1997) Retrieval of Old Memories: The Temporo-Frontal Hypothesis. Brain, 120, 1377-1399. http://dx.doi.org/10.1093/brain/120.8.1377

[12] Markowitsch, H.J. (1995) Which Brain Regions Are Critically Involved in the Retrieval of Old Episodic Memory? Brain Research Reviews, 21, 117-127. http://dx.doi.org/10.1016/0165-0173(95)00007-0

[13] Nyberg, L., McIntosh, A.R., Cabeza, R., Habib, R., Houle, S. and Tulving, E. (1996) General and Specific Brain Regions Involved in Encoding and Retrieval of Events: What, Where, and When. Proceedings of the National Academy of Sciences of the United States of America, 93, 11280-11285. http://dx.doi.org/10.1073/pnas.93.20.11280

[14] Tulving, E., Kapur, S., Craik, F.I.M., Moscovitch, M. and Houle, S. (1994) Hemispheric Encoding/Retrieval Asymmetry in Episodic Memory: Positron Emission Tomogtaphy Findings. Proceedings of the National Academy of Sciences of the United States of America, 91, 2016-2020. http://dx.doi.org/10.1073/pnas.91.6.2016

[15] Dimpfel, W., Koch, K. and Weiss, G. (2011) Early Effect of NEURAPAS ${ }^{\circledR}$ on Current Source Density (CSD) of Human EEG. BMC Psychiatry, 11, 123. http://dx.doi.org/10.1186/1471-244x-11-123

[16] Klimesch, W. (1997) EEG-Alpha Rhythms and Memory Processes. International Journal of Psychophysiology, 26, 319-340. http://dx.doi.org/10.1016/S0167-8760(97)00773-3

[17] Klimesch, W. (1999) EEG Alpha and Theta Oscillations Reflect Cognitive and Memory Performance: A Review and Analysis. Brain Research Reviews, 29, 169-195. http://dx.doi.org/10.1016/S0165-0173(98)00056-3

[18] Dimpfel, W. (2007) Characterization of Atypical Antipsychotic Drugs by a Late Decrease of Striatal Alpha1 Spectral Power in the Electropharmacogram of Freely Moving Rats. British Journal of Pharmacology, 152, 538-548. http://dx.doi.org/10.1038/sj.bjp.0707427

[19] Dimpfel, W. (2008) Pharmacological Modulation of Dopaminergic Brain Activity and Its Reflection in Spectral Frequencies of the Rat Electropharmacogram. Neuropsychobiology, 58, 178-186. http://dx.doi.org/10.1159/000191124 